\title{
Preface
}

\section{OrthoBiologics in Sports Medicine: Real-Time Applications Are Here, and Future Developments Are Promising!}

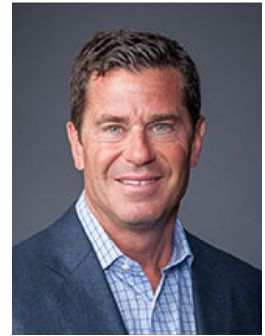

Brian J. Cole, MD, MBA

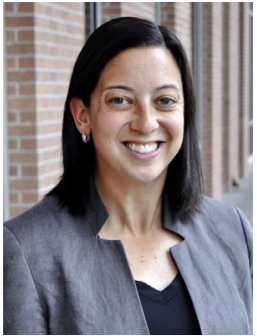

Rachel M. Frank, MD

Editors

OrthoBiologics have become increasingly utilized not only throughout the field of medicine but particularly in orthopedic surgery and sports medicine. The volume of literature describing products and techniques with a biologic basis has increased exponentially over the last several decades, with a variety of novel developments on the horizon. In this issue of Clinics in Sports Medicine we aim to bring our readers the most up-to-date information on OrthoBiologic techniques within sports medicine, utilizing a peer-reviewed format to ensure the information is accurate and reliable. While we hope that all literature can be thought of as accurate and reliable, this is of utmost importance when discussing novel biologic treatments, as the level of public misinformation is not insignificant. While many emerging therapies and techniques hold promise for improving function and reducing pain, a current product that has been proven to reproducibly regenerate damaged/diseased ligament, tendon, cartilage, and meniscus tissue, among other tissues, in a human population, unfortunately does not yet exist. Nevertheless, the field of OrthoBiologics is incredibly exciting, and the potential for tissue regeneration exists. In this issue of Clinics in Sports Medicine, multiple world-renowned experts summarize all available OrthoBiologic agents that have a role in the sports medicine patient population.

As many of the OrthoBiologic treatments that are described are administered via a local injection, Dr Kathy Weber begins this issue with a discussion of the most commonly utilized injection-based treatments for peri-articular and intra-articular conditions, including corticosteroid injections and viscosupplementation. $\mathrm{Dr}$ Jason Dragoo and colleagues then provide a comprehensive overview of platelet-rich 
plasma. From here, a more focused discussion of more novel OrthoBiologic treatments ensues, with Dr Andreas Gomoll and coauthors discussing the amnioticderived treatments and their typical utilization; Dr Elizaveta Kon and coauthors then summarizing adipose-derived stem cells; Dr Scott Rodeo and coauthors next discussing OrthoBiologics for bone healing; Dr Jorge Chahla and colleagues discussing OrthoBiologics for ligament repair and reconstruction; and Drs Rachel Frank and Brian Cole (your guest editors) and coauthors summarizing OrthoBiologics for focal chondral defects. Next, Dr Alan Getgood and colleagues describe the application of OrthoBiologics for the treatment of osteoarthritis, which, given the prevalence of osteoarthritis and its associated morbidity, will perhaps be one of the most clinically meaningful areas of OrthoBiologic application. Then, Dr Adam Anz and coauthors present emerging techniques and the future of OrthoBiologics in sports medicine, and finally, Dr Matthew Provencher and colleagues provide some real-life information on how to incorporate OrthoBiologics into your clinical practice. We hope that this collection of articles from this group of esteemed authors will prove useful for better understanding the real-time applications of OrthoBiologics in sports medicine, as well as the promising future developments being researched in basic, animal, translational, and clinical research studies around the globe.

Brian J. Cole, MD, MBA

Department of Orthopedics Department of Surgery, Rush OPH Midwest Orthopaedics at Rush Sports Medicine and Surgery Shoulder, Elbow and Knee Care Cartilage Restoration Center at Rush Rush University Medical Center

1611 West Harrison, Suite 300 Chicago, IL 60612, USA

Rachel M. Frank, MD Sports Medicine and Shoulder Surgery

Department of Orthopaedic Surgery University of Colorado School of Medicine

12631 East 17th Avenue Mail Stop B202

Aurora, CO 80045, USA

E-mail addresses:

bcole@rushortho.com (B.J. Cole) rachel.frank@ucdenver.edu (R.M. Frank)

Websites:

https://www.RachelFrankMD.com

http://www.BrianColeMD.com 